\title{
Generalized Transverse Resonance Analysis of Planar Discontinuities Considering the Edge Effect
}

\author{
Antoni Barlabé, Member, IEEE, Adolfo Comerón, Member, IEEE, and Lluís Pradell, Member, IEEE
}

\begin{abstract}
Mode-matching related techniques such as the Generalized Transverse Resonance Method (GTR) suffer from the relative convergence phenomenon. To reduce its influence, in this letter we present a technique consisting in the application of basis functions incorporating the singular behavior of fields at edges and an optimal modal ratio. We present the results obtained analyzing a uniform finline and a short-circuited finline which demonstrate the minimization of the relative convergence phenomenon, allowing a fast and accurate analysis on a low-cost personal computer.
\end{abstract}

Index Terms-Discontinuities, finline, generalized transverse resonance, numerical methods.

\section{INTRODUCTION}

A NALYSIS methods based upon the mode-matching technique suffer from the relative convergence phenomenon [1]: convergence to different solutions when increasing the number of modes for different modal ratios (ratio of the number of terms used to expand the fields in the different zones of the structure). Many studies on this topic have led to an optimum modal ratio [1], [2] or to an optimum matrix formulation in order to minimize the relative convergence phenomenon and/or to increase the rate of convergence for certain structures [3].

"A posteriori" tests such as the balance of complex power or plots of electric and magnetic fields at both sides of a discontinuity have been used to validate the results obtained and to recommend a particular formulation or modal ratio for the analysis of a particular structure [3]. The main objective of this work aims to establish the conditions that allow to be more confident "a priori" about the results that a particular method of analysis will give, specially when analyzing inhomogeneous structures such as finlines.

Use of the Generalized Transverse Resonance Technique (GTR) with Sorrentino's formulation for the analysis of uniform planar lines [4] [Fig. 1(a)], leads to an eigenvalue problem. The characteristic function is given by the determinant of a small-dimension symmetric real matrix, usually not ill-conditioned. Despite its good numerical behavior, the relative convergence phenomenon can still be observed (Fig. 2). The relative convergence problem is more severe in the case of discontinuities.

Manuscript received August 7, 2000; revised October 10, 2000. This work was supported by Spanish Government Grants 2FD97-0960-C05-05 and 2FD97-1769-C04-03 (CICYT - FEDER).

The authors are with the Universitat Politècnica de Catalunya, Dep. de Teoria del Senyal i Comunicacions, 08034 Barcelona, Spain.

Publisher Item Identifier S 1051-8207(00)11574-0.

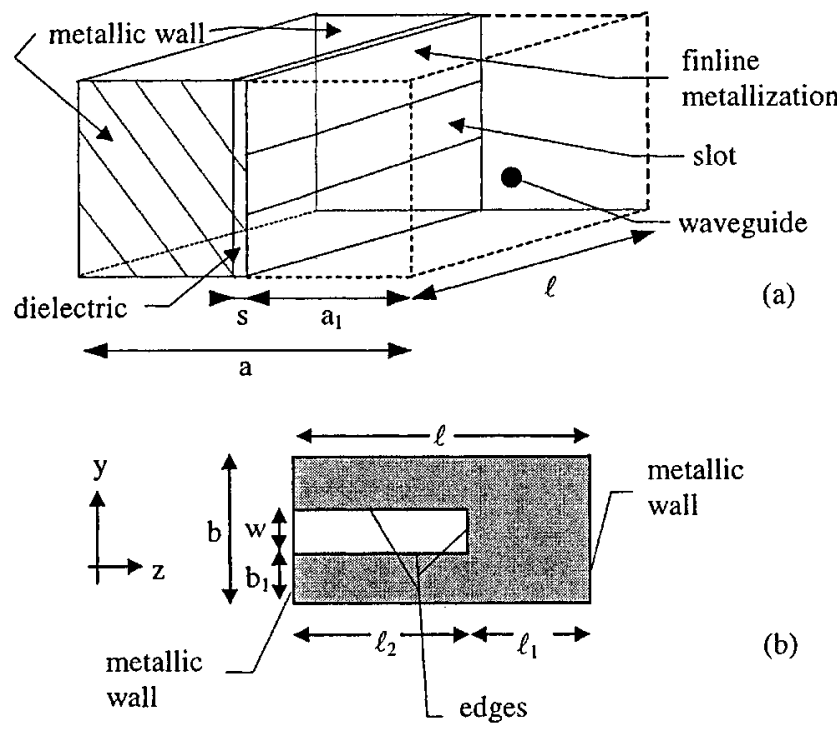

Fig. 1. (a) Finline in a rectangular resonant cavity for the application of GTR method. (b) Short-circuited finline.

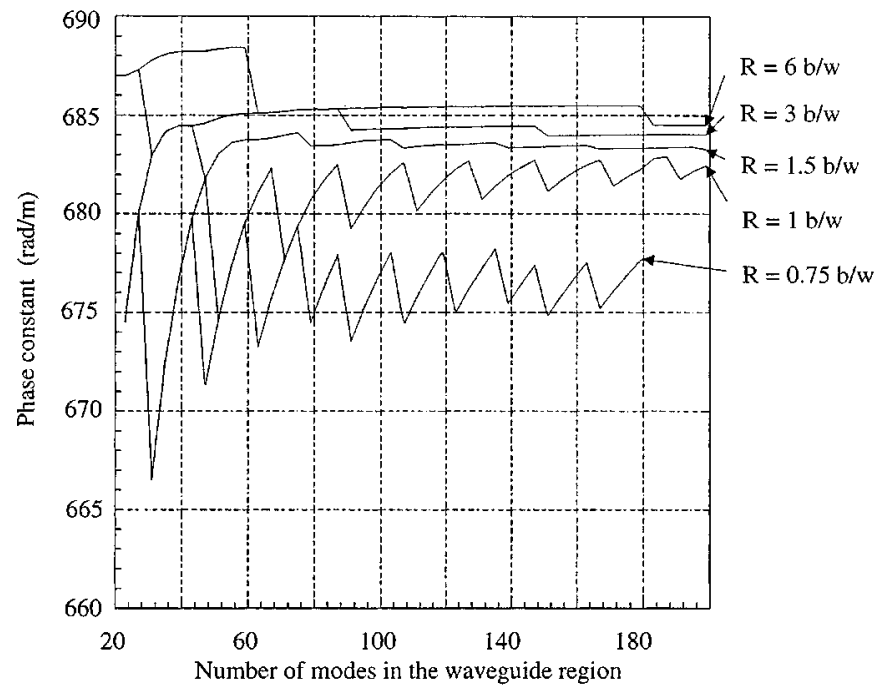

Fig. 2. Relative convergence phenomenon in the solution of the phase constant $(\beta)$ in a uniform unilateral finline for different modal ratios (number of modes in the waveguide region divided by the number of modes in the slot region) $R$. Dimensions as in Fig. 1. $a=7.11 \mathrm{~mm} ; b=a_{1}=3.56 \mathrm{~mm} ; w=0.71 \mathrm{~mm}$; $b_{1}=1.42 \mathrm{~mm} ; s=0.36 \mathrm{~mm} \varepsilon_{r}=2.2 ; f=31.6367 \mathrm{GHz}$.

\section{GTR FORMULATION INCLUDING THE EDGE CONDITION}

Application of the GTR technique to a planar structure enclosed in a rectangular waveguide [Fig. 1(a)] needs the evaluation 
TABLE I

Results of the AnAlysis of A Short-Ciruited Finline (SAME Dimensions ANd FreQuency AS IN Fig. 2)

\begin{tabular}{l|c|c}
\hline \multicolumn{1}{c|}{ Realization } & Equivalent normalized reactance & Phase of the reflection coefficient $\left(^{\circ}\right)$ \\
\hline Without considering & 0.20301 & 157.05 \\
$\mathrm{~W}_{\mathrm{y}}$ and $\mathrm{W}_{\mathrm{z}}$ & & \\
\hline Considering $\mathrm{W}_{\mathrm{y}}$ only & 0.20333 & 157.01 \\
\hline Considering $\mathrm{W}_{\mathrm{z}}$ only & 0.35211 & 141.21 \\
\hline Considering $\mathrm{W}_{\mathrm{y}}$ and $\mathrm{W}_{\mathrm{z}}$ & 0.34819 & 141.61 \\
\hline
\end{tabular}

of inner products $\mathrm{g}$ between the orthonormal modal functions of the waveguide $\vec{e}$ and those of the slot $\vec{e}_{0}[4]$

$$
g=\iint_{s} \vec{e} \cdot \vec{e}_{0} d s
$$

where $S$ is the surface of the slot enclosed in the rectangular cavity formed to use the GTR method.

If we modify the field expansions in the slot introducing an appropriate function taking into account the singular behavior of fields at edges

$$
\vec{e}_{0}^{\prime}=W \vec{e}_{0}
$$

which is equivalent to a generalization of the inner product

$$
g^{\prime}=\iint_{s} \vec{e} \cdot \vec{e}_{0}^{\prime} d s=\iint_{s} \vec{e} \cdot \vec{e}_{0} W d s
$$

the edge condition will be satisfied and the relative convergence phenomenon minimized.

For a uniform finline [Fig. 1(a)] we use the following weighting function because it satisfies the edge condition and its integration gives a closed analytical expression

$$
W=\frac{1}{\sqrt{1-\left(\frac{y-b_{1}-w / 2}{w / 2}\right)^{2}}} .
$$

The electric field at the slot is given by the modal functions of an empty rectangular waveguide of width $\ell$ and height $w$ modified by the weighting function $W$.

The electric field in the waveguide region is given by the transverse components of TE and TM modes in a rectangular empty waveguide of dimensions $\ell \times b$.

In general

$$
\begin{aligned}
g^{i} & =\int_{0}^{\ell} \int_{b_{1}}^{b_{1}+w} \vec{e}^{e, m} \cdot \vec{e}_{0}^{e, m} d y d z \\
& =f\left\{(m, n, p, q),\left(\ell, b_{1}, w, b\right), J_{0}\left[\left(\frac{n \pi}{b}+\frac{q \pi}{w}\right) \frac{w}{2}\right]\right\} .
\end{aligned}
$$

The inner products $g^{i}$ are real numbers which are function of the modal indices $(m, n, p, q)$, the physical dimensions $\left(l, b_{1}, w, b\right)$ and the Bessel function of first kind and order

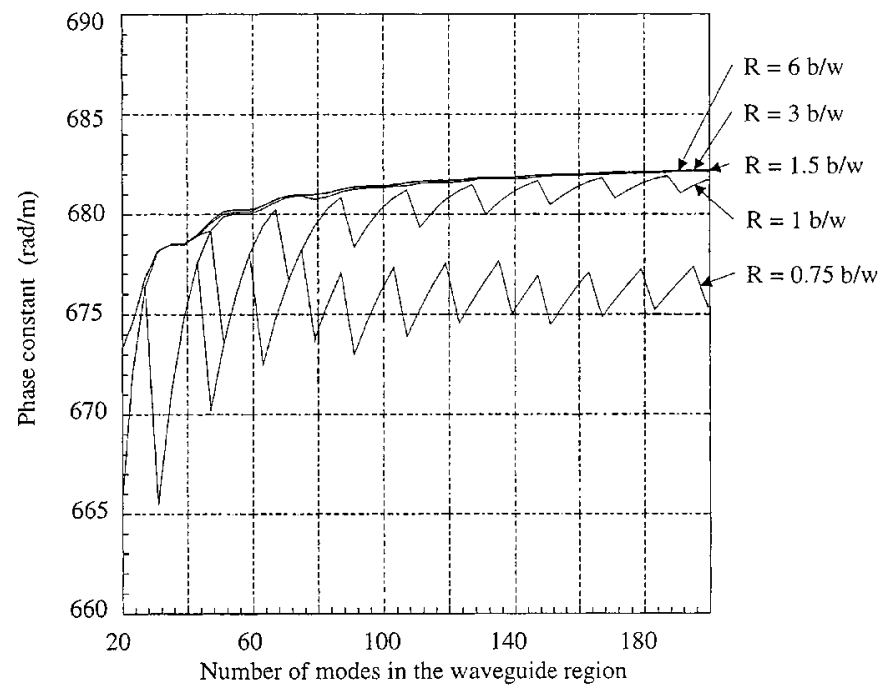

Fig. 3. Convergence of the solution when the edge effect is taken into account The mechanical dimensions and frequency are the same as in Fig. 2.

zero $\left(J_{0}(x)\right)$ (the superindex in the modal functions stands for TE or TM modes) [5].

\section{RESULTS}

\section{A. Analysis of a Uniform Finline}

Fig. 2 shows the results obtained when analyzing a uniform unilateral finline without taking into account the edge effect. Best results (as compared with those given by others methods such as the Spectral Domain Approach-SDA—or the Method of Lines-MOL-) are for a modal ratio $R$ of $1,5 \mathrm{~b} / \mathrm{w}$.

When the edge condition is incorporated in the basis functions it can be seen in Fig. 3 that a certain smooth convergence to the "true" (as predicted by other methods) value is obtained with

$$
R=\frac{N}{Q} \geq 1,5 \frac{b}{w}
$$

( $N$ is the number of modes in the waveguide region and $Q$ the number of modes in the slot).

The condition stated in (6) is an inequality, which means that is not necessary to determine an exact modal ratio for optimum convergence, simplifying the analysis method and adding confidence on the accuracy of the results obtained. 


\section{B. Analysis of a Short-Circuited Finline}

When analyzing a structure such as that depicted in Fig. 1(b), we have to cope with the problem of having two edges, so we use a double (separable) weighting function [5]

$$
W_{y}=\frac{1}{\sqrt{1-\left(\frac{y-b_{1}-w / 2}{w / 2}\right)^{2}}} ; \quad W_{z}=\frac{1}{\sqrt{1-\left(\frac{z}{\ell_{2}}\right)^{2}}}
$$

Then

$$
W=W_{y} W_{z} ; \quad \vec{e}_{0}^{\prime}=\vec{e}_{0} W=\vec{e}_{0} W_{y} W_{z} .
$$

Table I shows the comparison of the results obtained. It can be seen the relative importance of $W_{y}$ and $W_{z}$. The "higher" singularity of fields is in the finline short-circuit, making the inclusion of, at least $W_{z}$, necessary to obtain good results. For this case, Knorr [6] gives an equivalent normalized reactance of 0.35 .

For a good analysis it is enough taking 13 modes in the slot, which gives $8 \mathrm{~s}$ of CPU time on a PC-486-66 MHz computer (a 32-b low cost FORTRAN compiler was used).

\section{CONCLUSIONS}

The inclusion of modal functions taking into account the singular behavior of fields at edges and a non restrictive modal ratio condition in mode-matching related methods, such as the GTR, minimizes and even eliminates the relative convergence phenomenon, allowing accurate results with short modal expansions and fast evaluations on small, cheap personal computers. The technique presented can be applied with excellent results to the analysis of more complicated finline discontinuities such as steps and also to other planar discontinuities like inductive notches in coplanar waveguides.

\section{REFERENCES}

[1] R. Mittra and S. Lee, Analytical Techniques in the Theory of Guided Waves. New York: MacMillan, 1971.

[2] H. Hoffmann, Dispersion of Planar Waveguides for Millimeter-Wave Application: Arch. Elek. Ubert., Jan. 1977, vol. 31, pp. 40-44.

[3] Y. C. Shih, "The mode-matching method," in Numerical Techniques for Microwave and Millimeter-Wave Passive Structures, T. Itoh, Ed. New York: Wiley, 1989, ch. 9.

[4] R. Sorrentino, "Transverse resonance technique," in Numerical Techniques for Microwave and Millimeter-Wave Passive Structures, T. Itoh, Ed. New York: Wiley, 1989, ch. 11.

[5] A. Barlabé, "Anàlisi de discontinuïtats finline," Ph.D. dissertation, UPC, 1996.

[6] J. B. Knorr, "Equivalent reactance of a shorting septum in a fin-line: Theory and experiment," IEEE Trans. Microwave Theory Tech., vol. MTT-29, pp. 1196-1202, Nov. 1981. 